\title{
R600a 水平管内两相流型转换及摩擦压降特性
}

杨志强 ${ }^{1,2}$, 公茂琼 ${ }^{*}$, 陈高飞 ${ }^{1}$, 邹金金 ${ }^{1}$, 庄晓如 ${ }^{1,2}$, 宋庆路 ${ }^{1,2}$, 沈俊 ${ }^{1}$

1. 中国科学院理化技术研究所低温工程学重点实验室, 北京 100190;

2. 中国科学院大学, 北京 100049

* 联系人, E-mail: gongmq@ mail.ipc.ac.cn

2017-05-26 收稿, 2017-08-01 修回, 2017-08-01 接受, 2017-10-16 网络版发表 国家杰出青年科学基金(51625603)资助

摘要 R600a作为天然工质, 因其环境友好性和出色的制冷能力在欧美国家得到大范围推广, 被用作可替换制 冷剂之一. R600a两相流型转换和摩擦压降的精确预测对制冷系统的设计和优化具有重要参考意义。针对这个问 题, 开展了在内径为 $6 \mathrm{~mm}$ 的水平管中 $\mathrm{R} 600 \mathrm{a}$ 的两相流型以及摩擦压降的实验研究, 实验工况为饱和温度282.4 $304 \mathrm{~K}$, 质量流率范围 67 194 kg/( $\left.\mathrm{m}^{2} \mathrm{~s}\right)$. 实验中利用高速摄像仪, 观察到塞状流(plug flow)、层流波动流 (stratified-wavy flow)、弹状流(slug flow)、环状流(annular flow)4种流型, 并绘制流型图, 将流型图与文献中经典的 $I / A$ 流型转换曲线进行比较. 在综合考虑了气体惯性力、液体的黏性力和液体的表面张力对流型转换的影响, 引入 修正后的韦伯数 $W e^{*}$, 提出了新的流型转换曲线关联式. 同时基于环状流和非环状流的流型划分, 对摩擦压降分 区变化进行针对性的讨论, 发现摩擦压降在不同的流型区呈现出不同的变化规律. 将摩擦压降实验值与9种摩擦 压降关联式进行对比，结果显示Grönnerud关联式和Muller-Steinhagen \& Heck关联式分别能够较好预测非环状流 和环状流的两相摩擦压降.

关键词 R600a, 流型转换, 摩擦压降, 关联式

R600a作为自然工质, 因其环境友好性 $(\mathrm{ODP}=0$, GWP $<20)$ 和良好的热力性能被用做可替换的制冷剂 之一, 目前在家用电冰箱上有着广泛的应用 ${ }^{[1]}$. 而设 计和优化以 $\mathrm{R} 600 \mathrm{a}$ 为制冷剂的制冷系统需要可靠的 R600a两相流动数据作为支持. Ribatski和 Thome $\mathrm{e}^{[2]}$ 指 出要发展更高效率的紧凑热交换设备, 需要对传热 系数、压降、空隙率和流型进行可靠的预测. 而传热 系数、压降和空隙率又受到流型的影响, 因为不同的 流型会产生引起不同的气液相分布从而影响传热、压 降和空隙率. Rollmann和Spindler ${ }^{[3]}$ 同时也指出流型 的发展对压降的变化有着重要的影响. 两相摩擦压 降作为流体动力学特性之一, 是换热器、制冷系统、 热控系统等工业设备和系统设计与优化的重要参
数 ${ }^{[4]}$. 两相摩擦压降的精准预测不仅可以用来计算系 统动力输送特性, 同时也可以通过压力分布来确定 工质特性, 这对制冷系统的设计及优化起着重要作 用 ${ }^{[5]}$. 两相摩擦压降与两相流型的变化有着紧密的联 系, 所以对两相流动摩擦压降的精确预测同时需对 两相流型进行准确的判断. 因此近十几年来关于 R600a两相流动的特性研究也越来越受到关注.

针对 R600a在管内流动过程中两相流型和摩擦 压降变化特点、影响因素以及预测模型等问题，众多 学者们展开了一系列的相关研究. 针对R600a两相流 型图问题, Nasr等人 ${ }^{[6]}$ 在内径为 $8.7 \mathrm{~mm}$ 的水平光管中 开展了R600a流型实验研究. 实验结果表明在质量流 率130 380 kg/(m² s), 干度范围0 0.7, 热流密度 $10 \sim$

引用格式：杨志强, 公茂琼, 陈高飞, 等. R600a 水平管内两相流型转换及摩擦压降特性的实验. 科学通报, 2018, 63: 98-107 Yang Z Q, Gong M Q, Chen G F, et al. Experiment investigation of two-phase flow pattern transition and frictional pressure drop of R600a in a horizontal tube (in Chinese). Chin Sci Bull, 2018, 63: 98-107, doi: 10.1360/N972017-00587 
$27 \mathrm{~kW} / \mathrm{m}^{2}$ 的实验工况下，间歇流和环状流是主要流 型, 他们还将实验得出的流型图与经典流型图进行 对比, 结果显示Kattan等人 ${ }^{[7]}$ 的流型图能够较好预测 流型数据. 为研究环状流型区域内摩擦压降的变化 特性, Dalkilic等人 ${ }^{[8]}$ 在内径为 $4 \mathrm{~mm}$, 长度为 $1 \mathrm{~m}$ 的水 平铜管和内径为 $8.1 \mathrm{~mm}$, 长度为 $0.5 \mathrm{~m}$ 的坚直铜管中 分别开展了 R600a在环状流区域的流动冷凝摩擦压 降实验, 他们发现在环状流区域, 流动冷凝摩擦压降 与实验铜管的方向无关, 在知道管长的情况下, 可以 预测环状流区域的流动冷凝摩擦压降大小. Dalkilic 等人还将环状流区域流动冷凝摩擦压降数据与经典 关联式进行了对比, 结果表明Chen等人 ${ }^{[9]}$ 的关联式 可较好预测 R600a在环状流区域的流动冷凝摩擦压 降. 针对于不同管型和管径大小对摩擦压降所产生 的影响, Wen等人 ${ }^{[10]}$ 在内径为 $2.46 \mathrm{~mm}$ 的U型管内开 展R600a摩擦压降实验, 实验结果显示R600a摩擦压 降随着质量流率增大和U管的U型弯数量的增多而变 大. 2 个U型弯、 4 个U型弯和 6 个U型弯的U型管的摩擦 压降分别是直管的 1.26 倍、1.55倍和1.90倍. Lee和 $\mathrm{Son}^{[11]}$ 开展了 R600a在内径为 10.07 和 $5.80 \mathrm{~mm}$ 水平管 内流动冷凝传热和摩擦压降实验. 他们发现R600a制 冷剂在大管径中摩擦压降要小于小管径中的摩擦压 降, 并且在相同工况下, R600a在 10.7 和 $5.8 \mathrm{~mm}$ 管中 的摩擦压降比为6/15. 针对不同管壁对摩擦压降的影 响, Wen等人 ${ }^{[12]}$ 在 5 种具有不同管壁类型的管中开展 R600a摩擦压降实验研究, 他们发现摩擦压降随着管
壁上孔的数量和直径的增大而增大.

虽然近十几年来，学者们开展了不同工况下 R600a两相流动的实验研究, 但是很少有学者涉及 R600a的两相流型转换以及在不同流型区域内摩擦 压降特性的研究. 因此本文开展水平管内R600a两相 流动实验研究, 分析水平管内两相流型及不同流型 区域内摩擦压降的变化特性, 并探究适用于水平管 内R600a流型转换的关联式. 同时对不同流型区域内 摩擦压降与经典关联式进行了比较, 评估出各个流 型区域内预测精度较高的关联式.

\section{1 装置}

对R600a流型与压降特性的实验研究是在一套 自行设计的流动沸腾传热、压降以及流型特性的综合 实验台上完成的，该实验台是基于本课题组已有实 验台 ${ }^{[13]}$ 改进的. 实验装置如图1所示, 该系统分为测 试工质循环回路和制冷循环回路. 其中测试工质循 环回路包括传热测试段和绝热压降测试段, 绝热压 降测试段是一段长为 $1550 \mathrm{~mm}$, 内径为 $6 \mathrm{~mm}$, 外径 为 $8 \mathrm{~mm}$ 的铜管. 传热测试段是 $4 \mathbf{Z}_{\mathbf{Z}}$ 内径为 $6 \mathrm{~mm}$, 外 径 $30 \mathrm{~mm}$, 长度 $50 \mathrm{~mm}$ 紫铜加热段分别连接着内径为 $6 \mathrm{~mm}$, 外径为 $6.4 \mathrm{~mm}$ 的不锈钢管组成. 温度、压力及 压差传感器的具体布置情况如图1所示. 制冷循环回 路包括了主冷换热和过冷换热。测试工质在主冷换 热器中先被冷凝成液体, 再经过冷换热器进一步冷 却成过冷液体. 过冷工质通过一个可调速的磁力循

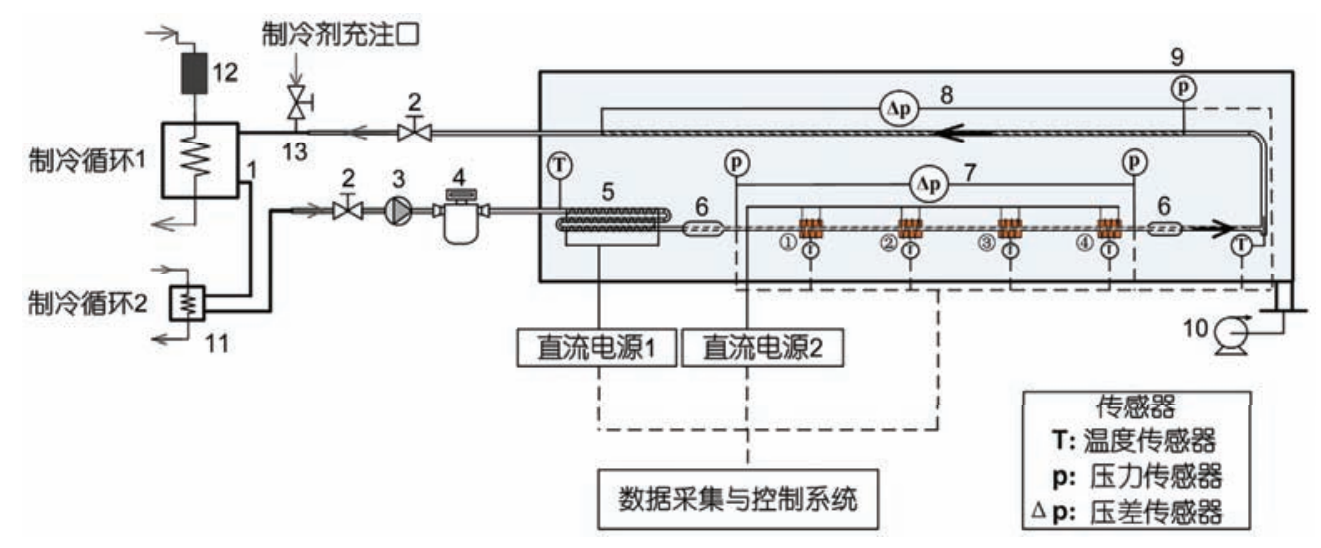

图 1 (网络版彩色)流动沸腾实验系统原理图. 1, 主换热器; 2 , 阀门; 3 , 磁力恶; 4 , 科氏流量计; 5 , 预热器; 6 , 观察窗; 7, 传热实验段; 8, 压降 实验段; 9 , 真空罩; 10 , 真空原; 11 , 过冷换热器; 12 , 加热棒; 13 , 制冷剂充注口

Figure 1 (Color online) Schematic view of flow boiling experimental system. 1, Heat exchanger; 2, valve; 3, magnetic gear pump; 4, coriolis mass flow meter; 5, preheater; 6 , sight glasses; 7 , heat transfer test section; 8, pressure drop test section; 9 , vacuum chamber; 10, vacuum pump; 11, heat exchanger; 12 , heating rod; 13 , refrierant charge port 
环泵驱动经预热段进行加热再进人测试段, 其质量 流率由一台科氏力质量流量计测得, 预热器主要用 于控制测试工质的人口干度. 为便于观察管内测试 工质的流动状态, 在传热测试段的进出口处均设置 了一个与测试段相同内径的石英玻璃管观察窗, 用 于直接观测管内两相流流型. 并且采用一台Motion Studio公司生产的Y4系列高速摄影仪, 其最高拍摄

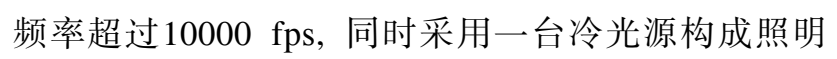
系统, 并辅助计算机采集系统完成实验过程中两相 流流型的观察与记录, 具体实物图可见图 2 .

\section{2 方法}

\section{1 管内两相流型特性}

本实验中将图 1 可视窗 6 (传热实验段出口处)出 现的流型作为绝热压降测试段 8 的人口流型, 观察到 塞状流(plug flow)、层流波动流(stratified-wavy flow)、 弹状流(slug flow)、环状流(annular flow)4种流型, 并且 将塞状流和弹状流合称为间歇流 (intermittent flow). 图3列出了一组由高速摄影仪记录的实际观测流型.

\section{2 管内两相流压降特性}

两相流的总压降由重力压降、加速压降和摩擦压 降3部分构成, 表达式为

$$
\Delta p_{\text {total }}=\Delta p_{\text {grav }}+\Delta p_{\text {mom }}+\Delta p_{\text {frict }},
$$

其中, $\Delta p_{\text {total }}$ 为管内流动总压降, 在实验中通过压差
传感器直接测得; $\Delta p_{\text {grav }}$ 为重力压降, 由重力势差引 起的, 本实验中实验段处于水平, 故 $\Delta p_{\mathrm{grav}}$ 为 $0 ; \Delta p_{\mathrm{mom}}$ 为加速压降, 由管内流体加速引起, 因本实验中压降 测试段为绝热状态, 无相变引起的加速, 故 $\Delta p_{\text {mom }}$ 为 $0 ; \Delta p_{\text {frict }}$ 为摩擦压降，由流体与壁面间的摩擦引起.

\section{3 干度的计算}

干度是实验过程中的重要参数. 实验中绝热摩 擦压降测试段的人口干度可由以下公式计算得到:

$$
\begin{aligned}
x_{\text {out }}= & \frac{Q_{\text {pre }}-C_{\text {pin }} m\left(T_{\text {in }}-T_{\text {sub }}\right)}{m H_{\mathrm{vv}-\mathrm{in}}}+\frac{Q_{1}}{m H_{\mathrm{vv}-1}}+\frac{Q_{2}}{m H_{\mathrm{lv}-2}} \\
& +\frac{Q_{3}}{m H_{\mathrm{v} v-3}}+\frac{Q_{4}}{m H_{\mathrm{lv}-4}},
\end{aligned}
$$

其中 $Q_{\text {pre }}$ 是预热段的加热量, $Q_{1}, Q_{2}, Q_{3}, Q_{4}$ 分别是传 热实验段中 4 个加热铜块的加热量, $T_{\text {in }}$ 是传热实验段 的人口温度, $T_{\mathrm{sub}}$ 是进人预热器前的过冷温度, $m$ 是质 量流量, $H_{\mathrm{IV}-\mathrm{in}}$ 是R600a在传热实验段人口的汽化潜热, $H_{\mathrm{lv}-1}, H_{\mathrm{Iv}-2}, H_{\mathrm{Iv}-3}, H_{\mathrm{Iv}-4}$ 分别是R600a在铜块1，2，3，4中 对应压力下的汽化潜热.

\section{4 实验不确定度分析}

实验中所使用的温度, 压力、压差、质量流率及 电压电流测量仪器的型号, 测量范围以及不确定度 如表1所示. 实验中对应的干度标准不确定度是通过 文献[14]中的计算方法得出, 在本文实验工况下, 通 过计算可得干度平均相对标准不确定度小于 $1.5 \%$ 。

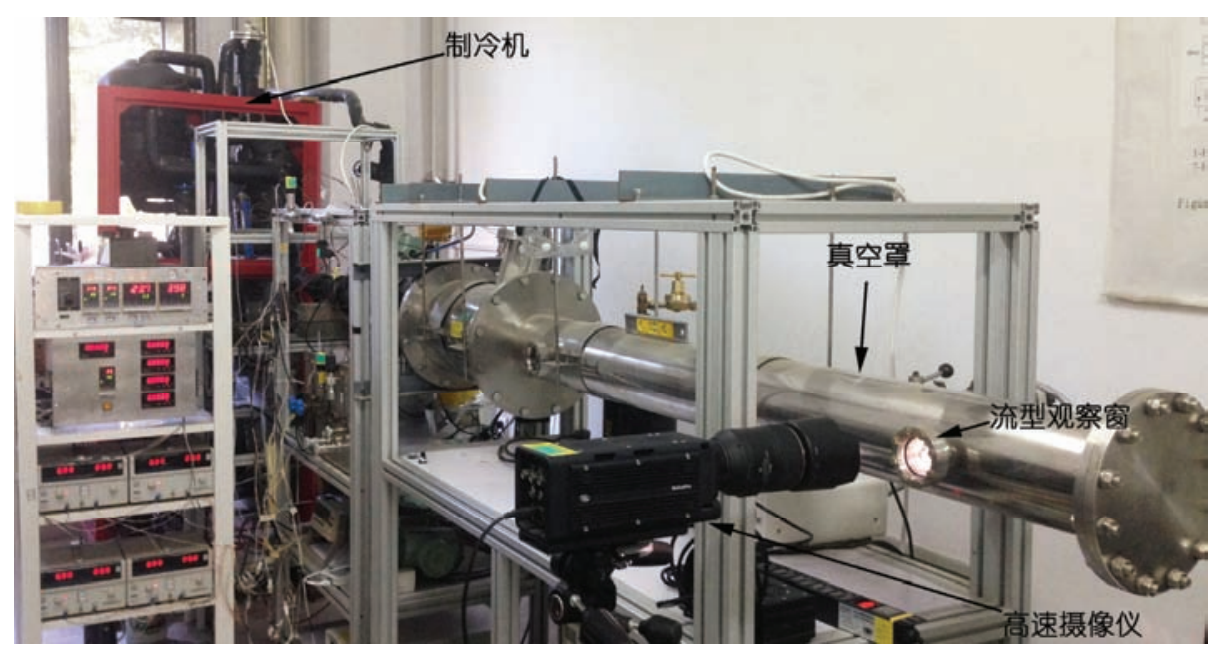

图 2 (网络版彩色) 流动沸腾实验系统实物图

Figure 2 (Color online) Physical experiment system of flow boiling 


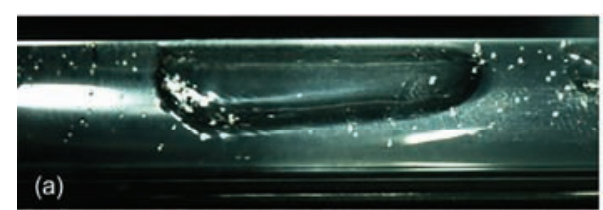

$p_{\text {ast }}=0.215 \mathrm{MPa}, G=129 \mathrm{~kg} /\left(\mathrm{m}^{2} \mathrm{~s}\right), q=10.6 \mathrm{~kW} / \mathrm{m}^{2}, x=0.0146$

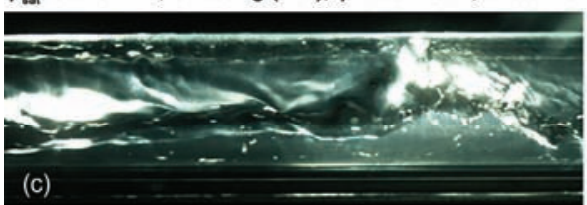

$P_{\text {sat }}=0.215 \mathrm{MPa}, \mathrm{G}=67 \mathrm{~kg} /\left(\mathrm{m}^{2} \mathrm{~s}\right), q=75.0 \mathrm{~kW} / \mathrm{m}^{2}, x=0.1290$

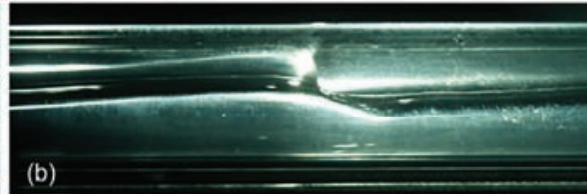

$P_{\text {att }}=0.215 \mathrm{MPa}, G=67 \mathrm{~kg} /\left(\mathrm{m}^{2} \mathrm{~s}\right), q=42.8 \mathrm{~kW} / \mathrm{m}^{2}, x=0.0150$

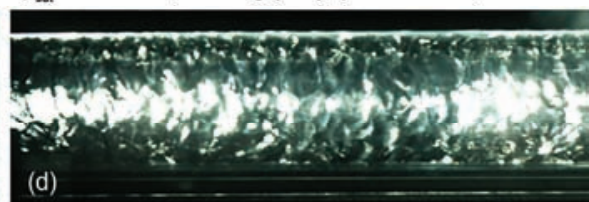

$P_{\text {sat }}=0.215 \mathrm{MPa}, G=67 \mathrm{~kg} /\left(\mathrm{m}^{2} \mathrm{~s}\right), q=75.0 \mathrm{~kW} / \mathrm{m}^{2}, x=0.5857$

图 3 (网络版彩色)实验中观察到的R600a流型图. (a) 塞状流; (b) 层流波状流; (c) 弹状流; (d) 环状流

Figure 3 (Color online) Photographs of R600a flow pattern. (a) Plug flow; (b) stratified-wavy flow; (c) slug flow; (d) annular flow

表 1 测量参数的不确定度

Table 1 Uncertainties of parameters

\begin{tabular}{llll}
\hline \multicolumn{1}{c}{ 参数 } & \multicolumn{1}{c}{ 仪器 } & \multicolumn{1}{c}{ 测量范围 } & \multicolumn{1}{c}{$\begin{array}{c}\text { 测量不 } \\
\text { 确定度 }\end{array}$} \\
\hline 温度 & PT100温度计 & $80 \sim 373 \mathrm{~K}$ & $\pm 0.1 \mathrm{~K}$ \\
压力 & GE UNIK 5000压力传感器 & $0 \sim 1 \mathrm{MPa}$ & $0.04 \%$ \\
压差 & GE UNIK 5000压差传感器 & $0 \sim 100 \mathrm{kPa}$ & $0.04 \%$ \\
质量流量 & ULTRA mass MKII科氏力 & & 质量流量计 \\
直流电压 & Keithley 2700数据采集器 & $0 \sim 60 \mathrm{~kg} / \mathrm{h}$ & $0.1 \%$ \\
电流 & ZW1659电流表 & $0 \sim 5 \mathrm{~A}$ & $0.005 \%$ \\
长度 & 游标卡尺 & $0 \sim 300 \mathrm{~mm}$ & $\pm 0.2 \%$ \\
\hline
\end{tabular}

\section{5 实验数据一致性验证分析}

为了验证实验数据的一致性, 本文在 R600a饱和 温度 $T_{\mathrm{sat}}$ 为 $282.4 \mathrm{~K}$, 质量流率 $G$ 为 $128 \mathrm{~kg} /\left(\mathrm{m}^{2} \mathrm{~s}\right)$ 的工况 下, 重复开展了 3 组在管内径为 $6 \mathrm{~mm}$ 的水平管内的压 降实验. 实验结果如图4所示. 在相同的工况下, 3组 实验所得摩擦压降数据之间偏差小于 $3.3 \%$, 偏差非

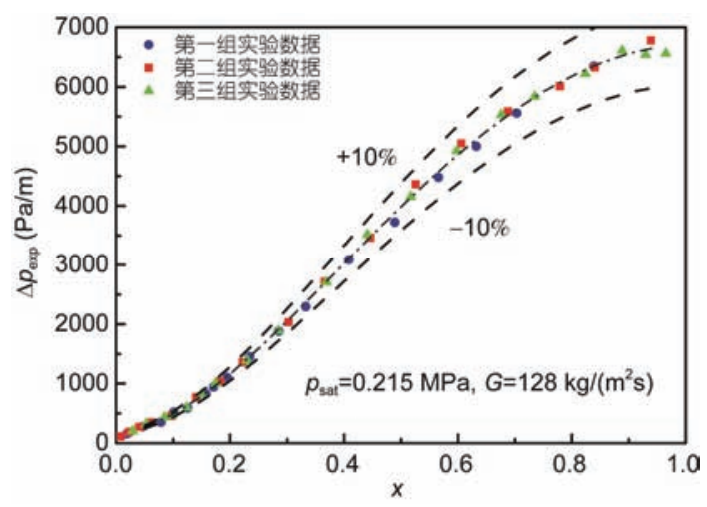

图 4 (网络版彩色)R600a在水平管内摩擦压降实验可靠性验证

Figure 4 (Color online) Reliability demonstration for frictional pressure drop of R600a
常小. 由图 4 可知, 3 组实验数据分布在一条曲线上, 一致性较好.

\section{3 结果与分析}

本文对 $\mathrm{R} 600 \mathrm{a}$ 在饱和温度 $T_{\mathrm{sat}}$ 为 $282.4,294.5$ 和 $304 \mathrm{~K}$, 质量流率 $G$ 范围为 $67 \sim 194 \mathrm{~kg} /\left(\mathrm{m}^{2} \mathrm{~s}\right)$, 干度 $x$ 范 围为 $0 \sim 1$, 管内径为 $6 \mathrm{~mm}$ 的水平管内压降以及流型 转换特性进行了实验研究.

\subsection{R600a两相流型图与经典流型转换曲线的比较}

图 5 为在饱和温度为 $282.4,294.5$ 和 $304 \mathrm{~K}$ 时 $\mathrm{R} 600 \mathrm{a}$ 在水平管内的流型数据与经典流型转换曲线 对比图, 其中 $I / A$ 指的是间歇流到环状流的转换曲线. 从图5分析可知, 在 3 组不同的质量流率 $(67,129,194$ $\left.\mathrm{kg} /\left(\mathrm{m}^{2} \mathrm{~s}\right)\right)$ 下, 流型的转变起始干度有所不同, 随着 质量流率的增大，间歇流向环状流转换的干度逐渐 减小. 在不同工况下环状流在所有流型中占比均最 高, 并且随着质量流率的增大, 环状流占比增大, 塞 状流(plug flow)、层流波动流(stratified-wavy flow)、弹 状流(slug flow)占比逐渐减小. 通过R600a两相流型 数据与经典的 $I / A$ 转换曲线的对比可知, 对于将 $I / A$ 转 换起始干度设定为固定值的Wojtan等人 ${ }^{[15]}$ 模型显然 与实验结果出人较大. 而Revellin等人 ${ }^{[16]}$ 的模型在预 测低质量流率的流型图时与实验实际情况偏差也非 常大. 虽然Barbieri等人 ${ }^{[17]}$ 和 Ong 等人 ${ }^{[18]}$ 的模型的 $I / A$ 转换预测趋势与流型实验数据较为接近, 但两者均 过度预测了 $I / A$ 转换时的起始干度值.

\section{2 新流型转换曲线的提出}

由图5分析可知，环状流占有的干度范围在所有 

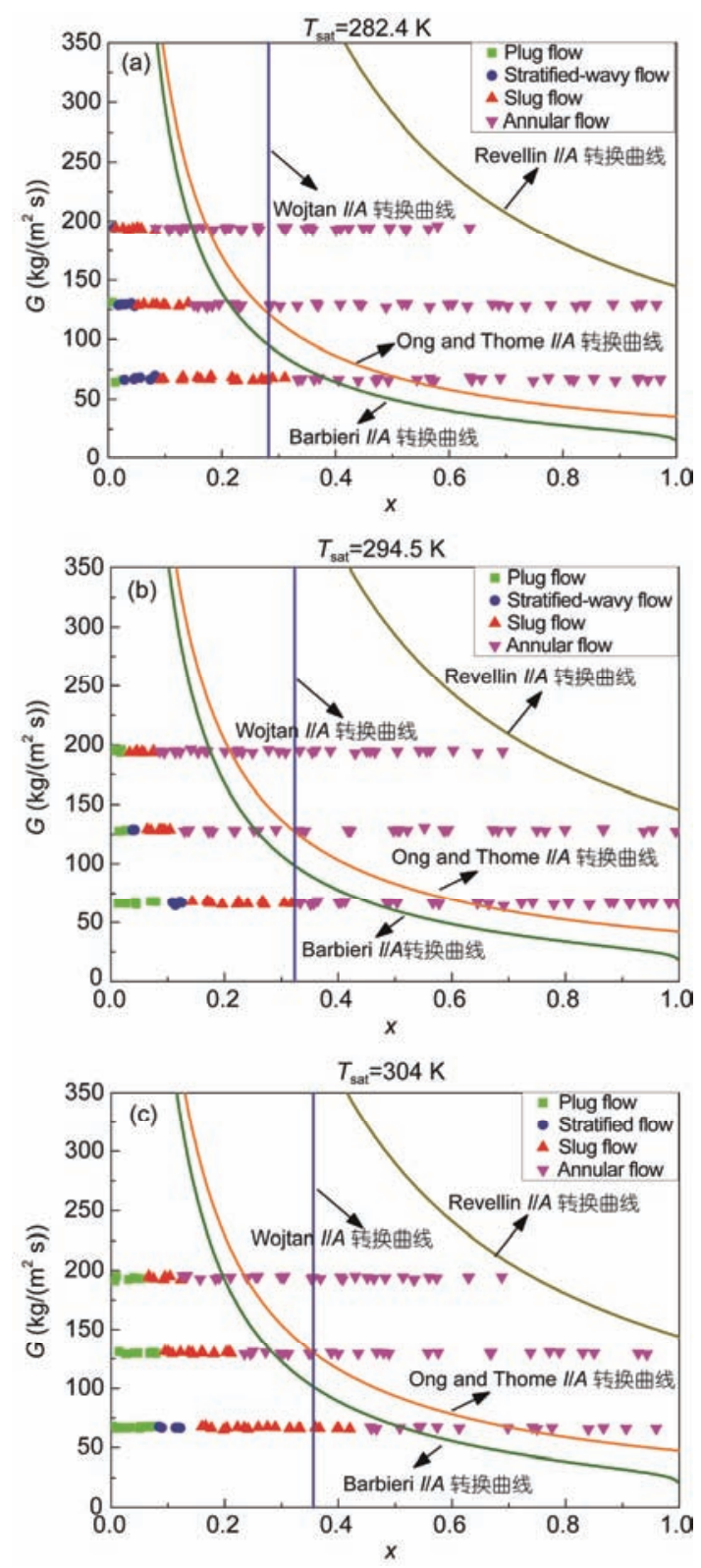

图 5 (网络版彩色) R600a在水平管内流型图与经典流型转换曲线对 比. (a) $T_{\text {sat }}=282.4 \mathrm{~K}$; (b) $T_{\text {sat }}=294.5 \mathrm{~K}$; (c) $T_{\text {sat }}=304 \mathrm{~K}$

Figure 5 (Color online) Flow pattern data for R600a compared with predicted $I / A$ transition models. (a) $T_{\text {sat }}=282.4 \mathrm{~K}$; (b) $T_{\text {sat }}=294.5 \mathrm{~K}$; (c) $T_{\text {sat }}=304 \mathrm{~K}$

流型中最大, 并且随着质量流率增大环状流在流型 中占比越高. Hewitt ${ }^{[19]}$ 和Ambrosini等人 ${ }^{[20]}$ 指出环状 流发生在广泛的工业设备和应用中, 比如核反应堆 堆芯、蒸汽发电机、再沸器、冷凝器、化工装置以及 石油和天然气井等, 是两相流型中最重要的流型. 因 此研究I/A转换曲线就显得尤为重要. Yang 和Shieh ${ }^{[21]}$, Chen等人 ${ }^{[22]}$, Zhang等人 ${ }^{[23]}$ 指出在管道中流动, 表面
张力对流型转换有重要的影响, 并且随着管径的减 小, 表面张力的影响逐渐增大. Rezkallah ${ }^{[24]}$ 和 Akbar 等人 ${ }^{[25]}$ 认为当流型从间歇流向环状流转换的过程中 黏性力和表面张力分别起着重要的作用. Zhuang等 人 ${ }^{[26]}$ 指出惯性力、黏性力以及表面张力对流型转换 有着重要影响, 并且基于这 3 个力对流型的影响开发 了新的流型转换关联式，该关联式能够很好预测流 型数据. 因此在综合考虑了惯性力、黏性力以及表面 张力对流型转换的作用, 本文引人一个由 Soliman ${ }^{[27]}$ 修正后的韦伯数 $W e^{*}$ 来描述气体惯性力、液体的黏性 力和液体的表面张力对流型转变的影响

$$
\begin{gathered}
W e^{*}=2.45 \frac{R e_{\mathrm{v}}^{0.64}}{S u_{\mathrm{v}}^{0.3}\left(1+1.09 X_{t t}^{0.039}\right)^{0.4}}, R e_{1} \leqslant 1250, \\
W e^{*}=0.85 \frac{R e_{\mathrm{v}}^{0.79} X_{t t}^{0.157}}{S u_{\mathrm{v}}^{0.3}\left(1+1.09 X_{t t}^{0.039}\right)^{0.4}}\left[\left(\frac{\mu_{\mathrm{v}}}{\mu_{1}}\right)^{2}\left(\frac{\rho_{1}}{\rho_{\mathrm{v}}}\right)\right]^{0.084}, \\
R e_{1}>1250, \\
S u_{\mathrm{v}}=\frac{\rho_{\mathrm{v}} \sigma D}{\mu_{\mathrm{v}}{ }^{2}} \\
R e_{1}=\frac{G(1-x) D}{\mu_{1}} \\
\operatorname{Re}_{\mathrm{v}}=\frac{G x D}{\mu_{\mathrm{v}}} \\
X_{t t}=\left(\frac{1-x}{x}\right)^{0.9}\left(\frac{\rho_{\mathrm{v}}}{\rho_{1}}\right)^{0.5}\left(\frac{\mu_{1}}{\mu_{\mathrm{v}}}\right)^{0.1}
\end{gathered}
$$

Kim 和Mudawar ${ }^{[28]}$ 发现在引人 $W e^{*}$ 后, 各流型之 间的转换关联式可以表达成 $W e^{*}=C X_{t t}^{n}$. 图6(d)表示的 是基于 $\mathrm{R} 600 \mathrm{a}$ 流型数据的 $W e^{*}$ 与 $X_{\mathrm{tt}}$ 之间关系图, 从图 中可知 $W e^{*}=C X_{t t}^{n}$ 这种关联式模型也可以很好地描述 R600a的 $I / A$ 流型转换. 通过对流型实验数据的拟合 可得R600a的 $I / A$ 转换关联式可以表达如下:

$$
W e^{*}=9.12 X_{t t}^{-0.02} \text {. }
$$

图 5(a) (c) 是新 $I / A$ 转换曲线与实验数据的对比 图, 从图中可知新的 $I / A$ 流型转换曲线可以很好地预 测流型转换的过程.

\section{3 基于流型的R600a摩擦压降分析}

图7所示在饱和温度分别为 $282.4,294.5,304 \mathrm{~K}$ 时, R600a在不同质量流率下两相摩擦压降随干度的 变化关系. 根据流型转换关联式将实验点分为非环 状流区域实验点和环状流区域实验点. 实验结果表 

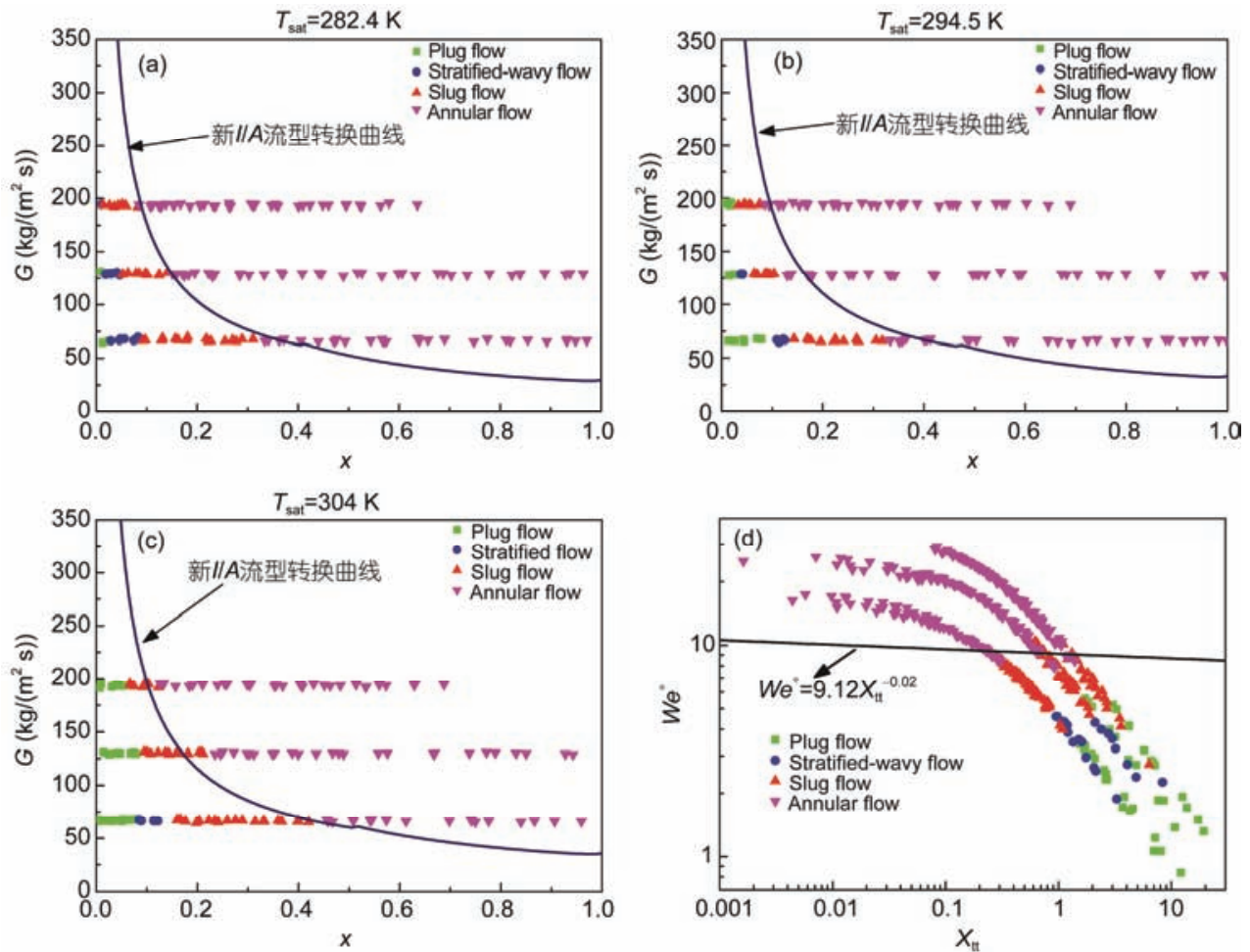

图 6 (网络版彩色)新 $I / A$ 转换曲线与R $600 \mathrm{a}$ 实验数据的对比图. (a) $T_{\mathrm{sat}}=282.4 \mathrm{~K}$ 时, 新 $I / A$ 转换曲线; (b) $T_{\mathrm{sat}}=294.5 \mathrm{~K}$ 时, 新 $I / A$ 转换曲线; (c) $T_{\mathrm{sat}}=304 \mathrm{~K}$ 时, 新 $I / A$ 转换曲线; (d) $T_{\mathrm{sat}}=282.4 / 294.5 / 304 \mathrm{~K}$ 时, 新 $I / A$ 转换曲线

Figure 6 (Color online) Flow pattern data for R600a compared with the new transition lines. (a) The new I/A transition line under $T_{\text {sat }}=282.4 \mathrm{~K}$; (b) the new $I / A$ transition line under $T_{\text {sat }}=294.5 \mathrm{~K}$; (c) the new $I / A$ transition line under $T_{\text {sat }}=304 \mathrm{~K}$; (d) the new $I / A$ transition line under $T_{\text {sat }}=282.4 / 294.5 / 304 \mathrm{~K}$
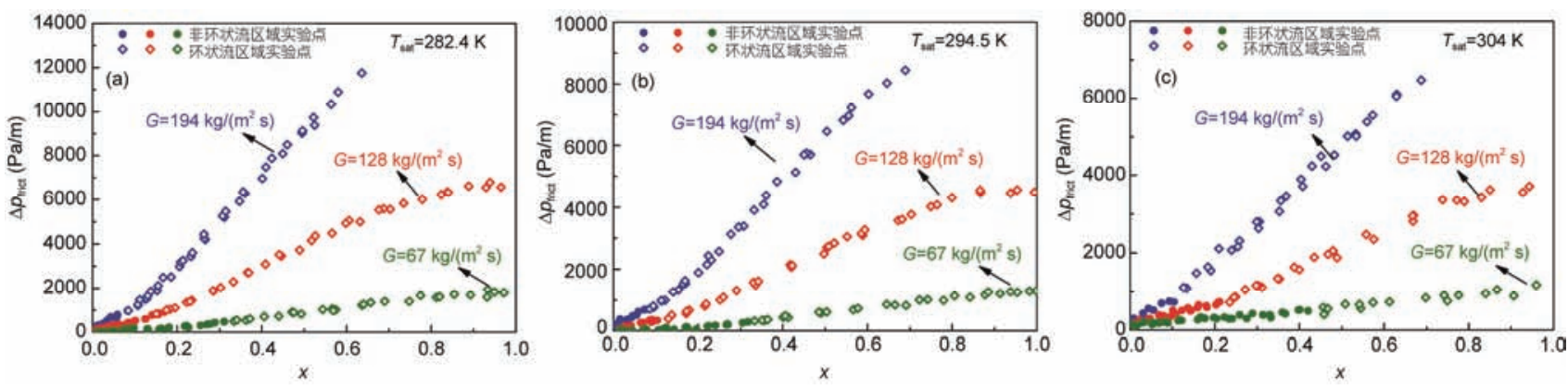

图 7 (网络版彩色)在不同质量流率时R600a摩擦压降随着干度的变化. (a) $T_{\mathrm{sat}}=282.4 \mathrm{~K}$; (b) $T_{\mathrm{sat}}=294.5 \mathrm{~K}$; (c) $T_{\mathrm{sat}}=304 \mathrm{~K}$

Figure 7 (Color online) Frictional pressure gradients for different mass fluxes. (a) $T_{\text {sat }}=282.4 \mathrm{~K}$; (b) $T_{\text {sat }}=294.5 \mathrm{~K}$; (c) $T_{\text {sat }}=304 \mathrm{~K}$

明，在非环状流区域和环状流区域时摩擦压降均随 着质量流率的增大而增大. 但是在不同流型区域内, 摩擦压降随着干度变化的趋势却有明显的不同. 当 两相流型处于非环状流区域时, 摩擦压降增长速度 缓慢, 当流型开始进入环状流区域时, 摩擦压降的增 长速度先明显加快然后减慢, 并最终趋于平缓. 这是 因为随着干度增大, 两相流速均增大, 而在环状流区 域, 空隙率较大, 由于气相压降对两相压降的影响占 主导地位, 气相流速越大, 摩擦压降越大. 以上分析
也表明了两相流型的改变对摩擦压降的变化产生明 显影响.

\section{4 不同流型区域摩擦压降实验数据与关联式 比较}

根据划分的流型区域，分别对在非环状流区域 和环状流区域的摩擦压降实验点进行分析，并将不 同流型区域的实验数据与 9 种摩擦压降关联式进行比

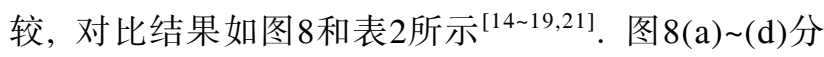



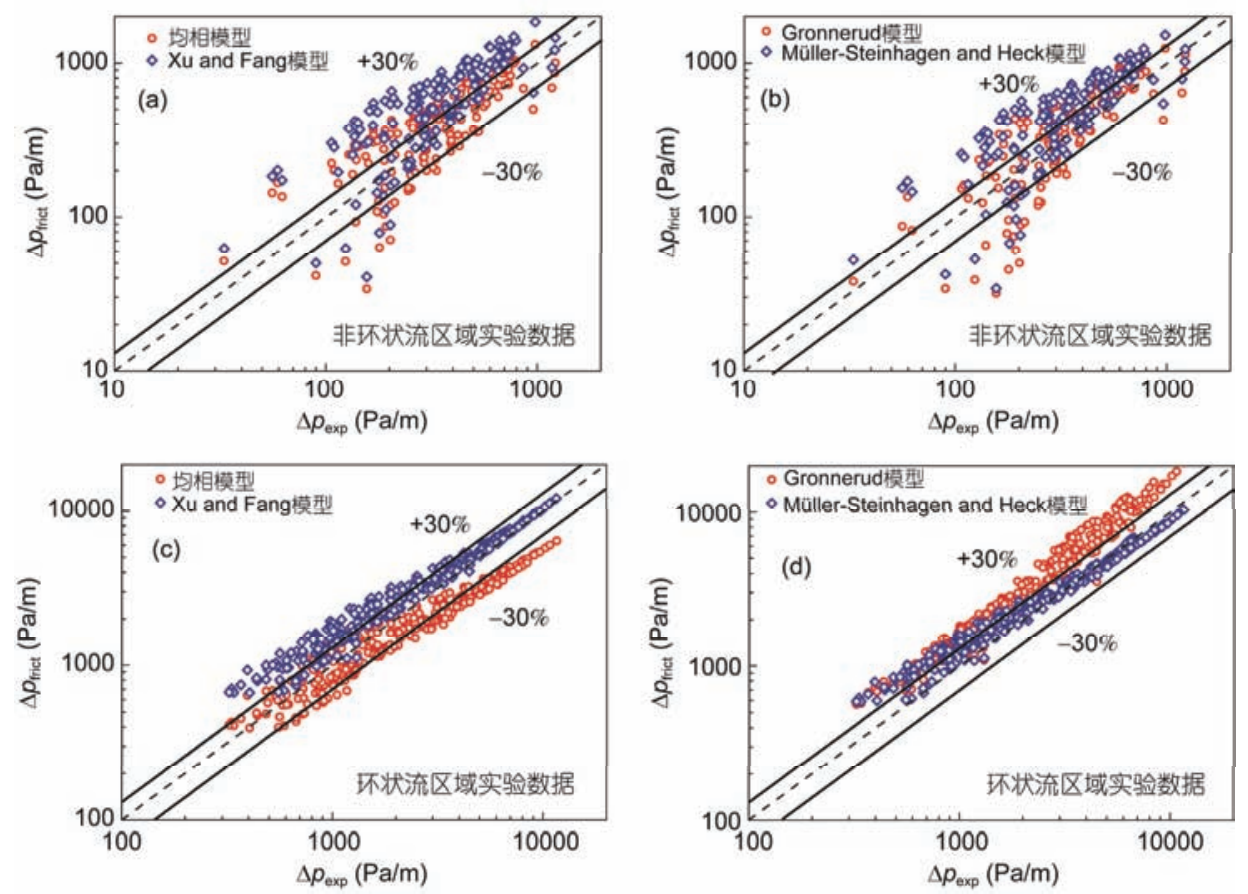

图 8 (网络版彩色)不同流型区域的摩擦压降实验值与经典关联式计算值对比. (a) 非环状流区域摩擦压降数据与均相模型和Xu \& Fang模型的 对比; (b) 非环状流区域摩擦压降数据与Grönnerud模型和Müller-Steinhagen \& Heck模型的对比; (c) 环状流区域摩擦压降数据与均相模型和Xu \& Fang模型的对比; (d) 环状流区域摩擦压降数据与Grönnerud模型和Müller-Steinhagen \& Heck模型的对比

Figure 8 (Color online) Comparison between predicted and experimental data of the frictional pressure drop. (a) The data of frictional pressure drop in non-annular region compare with Homogeneous model and Xu \& Fang model; (b) the data of frictional pressure drop in non-annular region compare with Grönnerud model and Müller-Steinhagen \& Heck model; (c) the data of frictional pressure drop in annular region compare with Homogeneous model and Xu \& Fang model; (d) the data of frictional pressure drop in annular region compare with Grönnerud model and Müller-Steinhagen \& Heck model

表 2 摩擦压降实验值与计算值的比较 ${ }^{a}$

Table 2 Deviations between calculated and measured frictional pressure drop

\begin{tabular}{|c|c|c|c|c|c|c|c|c|c|}
\hline \multirow{2}{*}{ 关联式 } & \multicolumn{3}{|c|}{ 非环状流区域数据 } & \multicolumn{3}{|c|}{ 环状流区域数据 } & \multicolumn{3}{|c|}{ 全部数据 } \\
\hline & MRD\% & MARD\% & $\lambda_{30 \%}$ & MRD\% & MARD\% & $\lambda_{30 \%}$ & MRD\% & MARD $\%$ & $\lambda_{30 \%}$ \\
\hline 均相模型 & 23.3 & 42.9 & 37.6 & -20.55 & 27.6 & 52.4 & -5.1 & 33.0 & 47.2 \\
\hline Lockhart \& Martinelli模型 ${ }^{[14]}$ & 159.9 & 162.3 & 4.7 & 47.1 & 64.6 & 34.8 & 86.9 & 99.1 & 24.2 \\
\hline Chisholm $^{[15]}$ & 287.4 & 288.4 & 2.7 & 127.5 & 129.6 & 16.9 & 184 & 185.7 & 11.8 \\
\hline Friedel模型[16] & 124.9 & 127.2 & 26.7 & 33.8 & 35.9 & 57.5 & 66.0 & 68.1 & 41.9 \\
\hline Grönnerud模型 ${ }^{[17]}$ & 17.3 & 38.5 & 44.3 & 57.1 & 57.4 & 11.0 & 43.1 & 50.8 & 22.7 \\
\hline Müller-Steinhagen \& Heck模型 ${ }^{[18]}$ & 45.8 & 57.1 & 32.2 & 15.8 & 19.7 & 75.8 & 26.4 & 32.9 & 60.4 \\
\hline Yan模型 ${ }^{[19]}$ & 170.1 & 170.5 & 3.4 & 10.8 & 42.6 & 41.0 & 67.0 & 87.8 & 27.7 \\
\hline $\mathrm{Xu}$ 和Fang模型 ${ }^{[1]}$ & 71.2 & 78.5 & 26.2 & 32.5 & 32.7 & 57.9 & 46.2 & 48.9 & 46.7 \\
\hline Kim和Mudawar模型 ${ }^{[21]}$ & 64.2 & 75.5 & 26.2 & -14.0 & 50.5 & 49.2 & 13.6 & 59.3 & 33.7 \\
\hline
\end{tabular}

a) $\mathrm{MRD}=$ 平均相对偏差 $=($ 实验值 - 预测值 $) /$ 实验值, $\mathrm{MARD}=$ 平均绝对偏差=|(实验值-预测值)/实验值|, $\lambda_{30 \%}$ 指平均相对偏差在 $30 \%$ 以 内的数据点占总数据点的百分比

别展示的是非环状流区域和环状流区域内摩擦压降 预测精度最好的 4 个经典模型. 从图 8 和表 2 可知, 经 典摩擦压降的关联式对环状流区域实验点的预测精
度比非环状流区域实验点高. 在非环状流区域, Grönnerud关联式预测效果最好, 其平均绝对偏差为 $38.5 \%$, 并且有 $44.3 \%$ 的实验数据在偏差 $30 \%$ 以, 均 
相模型预测效果次之, 但这两个关联式的预测值不 能均匀分布在实验值两侧, 尤其在低摩擦压降实验 值区域内, 预测值的分散程度较高. 在环状流区域 时, Muller-Steinhagen \& Heck关联式预测精度最高, 平均绝对偏差为 $19.7 \%$, 并且有 $75.8 \%$ 的实验数据偏 差在 $30 \%$ 以内, 均相模型预测效果次之. 在环状流 时, Muller-Steinhagen \& Heck模型预测值在低压降实 验值区域预测偏大而均相模型在此区域预测效果较 好, 但Muller-Steinhagen \& Heck模型在高压降实验 值区域预测值分布在实验值两侧, 预测效果较为准 确，而在此区域均相模型预测值明显偏小.

\section{4 结论}

本文开展了在内径为 $6 \mathrm{~mm}$ 的水平管中R600a的 两相流型转换以及摩擦压降的实验研究. 利用可视 化观测法, 观察到实验中存在塞状流、弹状流、层流 波动流和环状流 4 种流型. 并且对流型和摩擦压降数 据展开分析, 并得到以下结论:

(1) 分析R600a流型数据可知, 随着质量流率的 增大, 环状流转换起始干度逐渐减小. 在不同工况下 环状流在所有流型中干度区域占比最大, 并且随着
质量流率的增大，环状流占比逐渐增大. 通过与 4 种 经典流型转换曲线比较可知, Barbieri模型和 Ong 和 Thome模型的流型转换趋势与R600a流型转换趋势较 相似, 但两者均过度预测了环状流转换时的起始干 度值.

(2) 在流型转换模型中引人一个由Soliman修正 后的韦伯数 $W e^{*}$ 来描述气体惯性力、液体的黏性力和 液体的表面张力对流型转换的影响, 并提出了针对 $\mathrm{R} 600 \mathrm{a}$ 在水平管内新的 $I / A$ 流型转换关联式: $W e^{*}=$ $9.12 X_{t t}^{-0.02}$, 新流型转换关联式能够很好预测 $\mathrm{R} 600 \mathrm{a}$ 流 型转换.

(3) 随着质量流率和干度的增大, 摩擦压降逐渐 增大. 在不同的流型区域内摩擦压降的增长速度呈 现出不同的规律. 其中以进人环状流区域时摩擦压 降增长速度最快.

（4）基于新的流型转换关联式划分环状流区域 和非环状流区域, 并且将这两个流型区域内的两相 摩擦压降实验点与 9 种摩擦压降关联式进行对比, 结 果显示Müller-Steinhagen和Heck模型以及 Grönnerud 模型分别能够较好预测环状流区域和非环状流区域 的两相摩擦压降.

\section{参考文献}

1 Yan G, Cui C, Yu J. Energy and exergy analysis of zeotropic mixture R290/R600a vapor-compression refrigeration cycle with separation condensation. Int J Refrigeration, 2015, 53: 155-162

2 Ribatski G, Thome J R. Two-phase flow and heat transfer across horizontal tube bundles-A review. Heat Transfer Eng, 2007, 28: 508-524

3 Rollmann P, Spindler K. A new flow pattern map for flow boiling in microfin tubes. Int J Multiphas Flow, 2015, 72: 181-187

4 Xu Y, Fang X. A new correlation of two-phase frictional pressure drop for condensing flow in pipes. Nucl Eng Des, 2013, 263: 87-96

5 Wang S, Gong M Q, Chen G F, et al. Two-phase frictional pressure drop for propane in a horizontal tube (in Chinese). J Eng Thermophys, 2013, 34: 2155-2158 [汪胜, 公茂琼, 陈高飞, 等. 水平管内丙烷两相流动摩擦压降实验研究. 工程热物理学报, 2013, 34: 2155-2158]

6 Nasr M, Akhavan-Behabadi M A, Momenifar M R, et al. Heat transfer characteristic of R-600a during flow boiling inside horizontal plain tube. Int Commun Heat Mass, 2015, 66: 93-99

7 Kattan N, Thome J, Favrat D. Flow boiling in horizontal tubes: Part 1-development of a diabatic two-phase flow pattern map. J Heat Transf, 1998, 120: 140-147

8 Dalkilic A S, Agra O, Teke I, et al. Comparison of frictional pressure drop models during annular flow condensation of R600a in a horizontal tube at low mass flux and of R134a in a vertical tube at high mass flux. Int J Heat Mass Tran, 2010, 53: 2052-2064

9 Chen I Y, Yang K S, Chang Y J, et al. Two-phase pressure drop of air-water and R-410A in small horizontal tubes. Int J Multiphas Flow, 2001, 27: 1293-1299

10 Wen M Y, Ho C Y, Jang J K. Boiling heat transfer of refrigerant R-600a/R-290-oil mixtures in the serpentine small-diameter U-tubes. Appl Therm Eng, 2007, 27: 2353-2362

11 Lee H S, Son C H. Condensation heat transfer and pressure drop characteristics of R-290, R-600a, R-134a and R-22 in horizontal tubes. Heat Mass Transfer, 2010, 46: 571-584

12 Wen M Y, Jang K J, Ho C Y. The characteristics of boiling heat transfer and pressure drop of R-600a in a circular tube with porous inserts. Appl Therm Eng, 2014, 64: 348-357 
13 Wang S, Gong M Q, Chen G F, et al. Two-phase heat transfer and pressure drop of propane during saturated flow boiling inside a horizontal tube. Int J Refrigeration, 2014, 41: 200-209

14 Taylor B N, Kuyatt C E, Brown R H, et al. Guidelines for evaluating and expressing the uncertainty of NIST measurement results. NIST Tech Note 1297, Physics Laboratory, National Institute of Standards and Technology, 1994

15 Wojtan L, Ursenbacher T, Thome J R. Investigation of flow boiling in horizontal tubes: Part I-A new diabatic two-phase flow pattern map. Int J Heat Mass Tran, 2005, 48: 2955-2969

16 Revellin R, Dupont V, Ursenbacher T, et al. Characterization of diabatic two-phase flows in microchannels: Flow parameter results for $\mathrm{R}-134 \mathrm{a}$ in a $0.5 \mathrm{~mm}$ channel. Int J Multiphas Flow, 2006, 32: 755-774

17 Barbieri P, Jabardo J, Filho E B. Flow patterns in convective boiling of refrigerant R-134a in smooth tubes of several diameters. In: Proceedings of the 5th European Thermal-Sciences Conference. Eindhoven, 2008

18 Ong C L, Thome J R. Macro-to-microchannel transition in two-phase flow: Part 1-two-phase flow patterns and film thickness measurements. Exp Therm Fluid Sci, 2011, 35: 37-47

19 Hewitt G. Annular Two-Phase Flow. Pergamon: Elsevier, 1970

20 Ambrosini W, Andreussi P, Azzopardi B. A physically based correlation for drop size in annular flow. Int J Multiphas Flow, 1991, 17: 497-507

21 Yang C Y, Shieh C C. Flow pattern of air-water and two-phase R-134a in small circular tubes. Int J Multiphas Flow, 2001, 27: $1163-1177$

22 Chen L, Tian Y S, Karayiannis T G. The effect of tube diameter on vertical two-phase flow regimes in small tubes. Int J Heat Mass Tran, 2006, 49: 4220-4230

23 Zhang T, Cao B, Fan Y, et al. Gas-liquid flow in circular microchannel-Part I: Influence of liquid physical properties and channel diameter on flow patterns. Chem Eng Sci, 2011, 66: 5791-5803

24 Rezkallah K. Weber number based flow-pattern maps for liquid-gas flows at microgravity. Int J Multiphas Flow, 1996, 22: 1265-1270

25 Akbar M K, Plummer D A, Ghiaasiaan S M. On gas-liquid two-phase flow regimes in microchannels. Int J Multiphas Flow, 2003, 29: $855-865$

26 Zhuang X, Gong M, Chen G, et al. Two-phase flow pattern map for R170 in a horizontal smooth tube. Int J Heat Mass Tran, 2016, 102: $1141-1149$

27 Soliman $\mathrm{H}$. The mist-annular transition during condensation and its influence on the heat transfer mechanism. Int J Multiphas Flow, 1986, 12: 277-288

28 Kim S M, Mudawar I. Flow condensation in parallel micro-channels-Part 2: Heat transfer results and correlation technique. Int J Heat Mass Tran, 2012, 55: 984-994 


\title{
Experiment investigation of two-phase flow pattern transition and frictional pressure drop of R600a in a horizontal tube
}

\author{
Zhiqiang Yang ${ }^{1,2}$, Maoqiong Gong ${ }^{1 *}$, Gaofei Chen ${ }^{1}$, Xin Zou ${ }^{1}$, Xiaoru Zhuang ${ }^{1,2}$, \\ Qinglu Song ${ }^{1,2}$ \& Jun Shen ${ }^{1}$ \\ ${ }^{1}$ Key Laboratory of Cryogenics, Technical Institute of Physics and Chemistry, Chinese Academy of Sciences, Beijing 100190, China; \\ ${ }^{2}$ University of Chinese Academy of Sciences, Beijing 100049, China \\ * Corresponding author, E-mail: gongmq@mail.ipc.ac.cn
}

With the growing concern of environment problems, there are urgent demands for environment-friendly refrigerants in the refrigeration industry. As a natural refrigerant, R600a not only has zero ozone depletion potential and quite low global warming potential, but also has better cooling performance than other refrigerants. Due to its good cooling performance and environment-friendly characteristics, R600a has been used as an alternative refrigerant in heat transfer applications such as refrigerators, freezers, and heat pumps. It is known that accurate prediction of two phase flow pattern transition and frictional pressure drop of R600a in a horizontal tube play an important role in design and optimization of refrigerators, freezers, and heat pumps. According to this issue, a detailed experimental study was carried out to investigate the two-phase flow pattern transition and frictional pressure drop characteristics of R600a in a smooth horizontal tube with an inner diameter of $6 \mathrm{~mm}$. The experiments were performed at conditions covering saturation temperature from 282.4 to $304 \mathrm{~K}$, mass fluxes from 67 to $194 \mathrm{~kg} /\left(\mathrm{m}^{2} \mathrm{~s}\right)$. Based on a high speed camera, four main flow regimes can be observed: plug flow, stratified-wavy flow, slug flow and annular flow. The flow map of R600a has been drawn and comparisons between the experimental data of flow patterns and transition lines in the literature have been made. The results showed that with the increase of mass flux, the inception of the annular flow regime occurs earlier. The intermittent flow regime takes place over a narrow range of vapor quality and annular flow regime occupies a wide range of vapor quality. In addition, the trend of Barbieri and Ong and Thome I/A transitions lines are consistent with the experimental data. However, both of them overestimate the inception of the annular flow regime. After taking the influence of vapor inertia, liquid viscous and surface tension forces into account, a modified Weber number $W e^{*}$ has been introduced to the new flow pattern transition model. Based on the relationship of $X_{t t}$ and $W e^{*}$, a new correlation has been proposed and it can well fit with the experimental data of flow pattern. Furthermore, a detailed discuss of frictional pressure drop of annular flow and non-annular flow has been made. The results indicated that frictional pressure drop increases with the increases of vapor quality. The tendency of frictional pressure drop presents different the changing laws in different flow regime. The frictional pressure drop increases slowly in the non-annular flow regime while it increases rapidly when the flow pattern turns into the annular flow regime. The reason is that the vapor-phase pressure drop often dominates the total two-phase frictional pressure drop. The annular flow regime owns higher void fraction than the non-annular flow regime. Nine classic prediction correlations of pressure drop were evaluated and the results showed that the correlation of Grönnerud and Muller-Steinhagen and Heck give the best fit to the experimental frictional pressure drop of annular flow and non-annular flow with a mean absolute relative deviation of $38.5 \%$ and $19.7 \%$ respectively.

R600a, flow pattern transition, friction pressure drop, correlation

doi: 10.1360/N972017-00587 